\title{
Recurrent oligometastatic transitional cell bladder carcinoma: is there room for radiotherapy?
}

\author{
M. AUGUGLIARO ${ }^{1,2}$, G. MARVASO ${ }^{1}$, D. CIARDO ${ }^{1, *}$, D. ZERINI', G. RIVA ${ }^{1,2}$, E. RONDI ${ }^{3}$, S. VIGORITO ${ }^{3}$, S. COMI ${ }^{3}$, O. DE COBELLI ${ }^{2,4}$, R. ORECCHIA ${ }^{5,6}$, \\ B. A. JERECZEK-FOSSA ${ }^{1,2}$ \\ ${ }^{1}$ Department of Radiotherapy, European Institute of Oncology, Milan, Italy; ${ }^{2}$ Department of Oncology and Hemato-oncology, University of \\ Milan, Milan, Italy; ${ }^{3}$ Unit of Medical Physics, European Institute of Oncology, Milan, Italy; ${ }^{4}$ Department of Urology, European Institute of Oncol- \\ ogy, Milan, Italy; ${ }^{5}$ Scientific Direction, European Institute of Oncology, Milan, Italy; ${ }^{6}$ Department of Medical Imaging and Radiation Sciences, \\ European Institute of Oncology, Milan, Italy \\ ${ }^{*}$ Correspondence: delia.ciardo@ieo.it
}

Received May 22, 2018 / Accepted July 4, 2018

\begin{abstract}
This paper presents a retrospective report on radiotherapy (RT) in the oligometastastic recurrence of bladder cancer. Thirteen patients treated for low-volume metastatic transitional cell urinary bladder carcinoma (TCC) were reviewed, with the primary endpoint to evaluate the safety and efficacy of RT, proposed as an alternative to systemic treatment and/or to defer commencement of systemic therapy. The inclusion criteria were: patients who received RT without other local/ systemic therapy for oligometastatic TCC with lymph node, bone and lung lesions or local recurrence. Previous systemic therapy and surgery on the primary tumor were allowed in this tumor response, and toxicity evaluation and progression free-survival was also assessed. Thirteen patients with 21 lesions were treated with stereotactic body radiotherapy (SBRT) or conformal 3D radiotherapy (3D-CRT) between 2012 and 2017. All participants were discussed by a multidisciplinary urological board. The median age at RT was 68 years (range 50-80), the median Karnofsky performance status (KPS) was 90 (range 80-90) and the median interval between TCC diagnosis and commencement of RT on oligometastasis was 23 months (range 8-105). The median treatment dose was $25 \mathrm{~Gy}$ (range 20-36 Gy) given over a median of 5 fractions (range 3-10 fractions) with a median follow-up of 25 months (range 3-43 months). Imaging assessment was available for 20 lesions. The radiological progression of disease was registered in 9 patients at the median of 4.2 months from radiotherapy (range 1.9-18.8 months). This identified in-field and out-field progression in 6 patients and only out-field progression in the remaining 3. At last contact, 3 patients were alive with no evidence of disease, 3 had evidence of disease, 6 died of cancerrelated disease and one died from another cause. No severe acute and late toxicity was observed.

The literature contains no consistent data on TCC oligometastatic setting, but radiotherapy on lymph node, bone and/or lung oligo-recurrence from TCC offers durable disease control in a small number of patients with a very low toxicity profile. Further studies are required to establish the radiotherapy role in oligometastatic recurrent bladder cancer.
\end{abstract}

Key words: external beam radiotherapy, stereotactic body radiotherapy, oligometastatic bladder cancer

Metastatic disease remains the leading cause of death in cancer patients. This is particularly evident in patients with metastatic bladder cancer, where the prognosis is poor because of the aggressive nature of the tumor.

About $10-15 \%$ of patients present with metastatic disease at diagnosis, while $50 \%$ of patients undergoing radical cystectomy for invasive bladder cancer develop pelvic or distant recurrence [1]. The probability of developing lymph node metastases of a tumor with muscular wall infiltration is $15-25 \%$. The survival for patients with lymph node metastases at the time of surgery might be from 6 months to 2 years [2].
Recurrence depends on both the stage and the nodal status. In high-grade tumors and in advanced stages (pT3b-4), lymph node involvement $(\mathrm{pN}+)$ is frequently observed (40-60\% of cases) during radical cystectomy [3]. Although the 5-year survival for patients with muscle invasive bladder cancer with no nodal involvement may reach $60 \%$, these figures are much lower for pT3-pT4, pN1-3, M0/M1 cases (5-year survival after radical cystectomy is equal to $5-30 \%$ ) $[4,5]$.

In metastatic patients, chemotherapy is the treatment of choice and plays a palliative role. The most commonly used schedules include gemcitabine and cisplatin or methotrexate, 
vinblastine, doxorubicin, cisplatin (M-VAC) schemes that showed no significant differences in survival [6], but only in terms of tolerability. More recently, new studies showed the efficacy of immune-check point inhibitors in advanced bladder cancer [7-9]. Systemic therapy remains the cornerstone of metastatic bladder cancer, however, not every patient is fit enough to undergo such treatment. The mean age at the diagnosis of metastatic bladder cancer is $>65$ years old and due to the smoking-based etiology of bladder cancer [10], the majority of patients present severe smoking-related comorbidity. Therefore, less invasive approaches could be of great value in this particular cancer patient population. This can be of special concern in the patients with limited metastatic cancer, so called oligometastatic patients (i.e. patients with number of lymph nodes, bone or lung metastases less or equal to 5, based on the current definition of oligometastatic disease).

Metastatic cancer is a wide range of the disease, including both patients with single bone or soft tissues (lymph node) metastasis and widespread visceral dissemination. In particular, the unfit patients with limited metastatic disease might benefit from local approach like stereotactic irradiation. Stereotactic body radiotherapy (SBRT) has been studied in numerous settings including oligometastatic lung, colorectal, prostate and breast cancer [11-17]. Only limited data are available for SBRT in oligometastatic bladder cancer, including mainly case reports and series of SBRT for primary transitional cell carcinoma (TCC) and renal cell carcinoma of kidney [18-21].

The aim of our retrospective study was to review the data on tumor outcome and toxicity profile in a series of 13 patients treated with RT for TCC with lymph node, bone, lung or local oligorecurrence, at the high-volume comprehensive cancer center.

\section{Patients and methods}

The study was part of the research on SBRT notified to Ethics Committee of IRCSS European Institute of Oncology and Centro Cardiologico Monzino (Via Ripamonti, 435, 20141 Milano, Italy) (notification Nr. 93/11).

Study population. We retrospectively reviewed data of patients following these inclusion criteria:

1. histological diagnosis of TCC;

2. documented stage IV (N1-3/M1) disease at the time RT, based on the American Joint Committee on Cancer (AJCC) TNM stage;

3. any kind of previous TCC therapy was permitted (cystectomy and systemic therapy or cystectomy alone);

4. number of lymph node, bone or lung metastases less or equal to 5 (based on the current definition of oligometastatic disease);

5. Karnofsky performance status $\geq 80$ at the time of RT, i.e. fit enough to receive cytoreductive RT (not just palliation);

6. life expectancy of at least 3 months;
7. no concomitant systemic therapy during RT;

8. written informed consent for the RT;

9. written informed consent for use of the anonymized data for research and educational purpose.

The diagnosis of a clinically evident nodal, bone, lung metastases or local recurrence of TCC was based on imaging studies. Total body staging was required to exclude other disease sites, using a computer tomography (CT) scan of the thorax and abdomen and a bone scan and/or 18F-Fluorodeoxyglucose positron emission tomography/CT $\left(\left[{ }^{18} \mathrm{~F}\right]-\mathrm{FDG}\right.$ PET/CT). Lymph nodes were classified as "pelvic" or regional $(\mathrm{N})$ and "extrapelvic" or metastatic (M). The indication to RT for the recurrent disease was discussed in our multidisciplinary tumor board.

Patients treatment. We included patients with previous different treatments. Specifically, cystectomy and pelvic lymphadenectomy was performed in 12 patients. Seven patients were treated with Cisplatin and Gemcitabine (CDDP/GEM) for 4 cycles as adjuvant therapy, one of whom performed a second CDDP/GEM therapy for local disease recurrence and distant metastasis. One patient underwent two lung lobectomies for TCC metastases before being subjected to SBRT for other lung disease localization. Cystectomy is not performed in one patient because of advanced age, associated comorbidity or patient refusal. RT to oligometastasis was proposed in order to postpone cancer progression, and defer other treatments, specifically chemotherapy in patients with advanced age, comorbidity especially cardiovascular or renal insufficiency, previous surgery (in the case of SBRT to lung metastasis).

Follow-up procedure and definition of progression. After RT, clinical and radiological examinations were performed every 3-4 months. The local response to treatment (response of the treated lesion) was scored as a complete response (CR), partial response (PR), stable disease (SD), or progressive disease (PD) according to Response Evaluation Criteria In Solid Tumors (RECIST) criteria [22].

Clinical progression was defined as documented local progression, regional or systemic disease. Clinical progression was also classified as in-field progression, defined as the documented clinically and radiologically failure in the RT field, and out-field progression, intended as the relapse outside the RT field (i.e. distant progression). Progression free survival (PFS) was defined as the time from the beginning of RT to the time of disease progression. Gastrointestinal (GI) and genitourinary (GU) toxicity was recorded using Radiation Therapy Oncology Group/European Organization for Research and Treatment of Cancer (RTOG/EORTC) criteria [23]. Acute toxicity was analyzed in all patients. Late toxicity was evaluated in the patients with a minimum 6-month follow-up.

Compliance with the ethical standards. In this research, no animals were involved. All patients signed a written informed consent for stereotactic body radiation therapy (SBRT) and written informed consent for the use of the 
anonymized data for research or educational purpose. The study was performed within the Institutional Ethics

Table 1. Patient and lesion characteristics.

\begin{tabular}{lc}
\hline Characteristics & 68 (50-84) \\
\hline Age at the first RT [years], Median (range) & 13 \\
Histology & \\
TCC & 12 \\
Previous RT treatment & 7 \\
$\quad$ Previous surgery & 2 \\
Previous systematic chemotherapy & 1 \\
Other surgery (lung lobectomy) & \\
No surgery & 1 \\
KPS at the RT & 12 \\
80 & \\
90 & \\
Treated lesions & 8 \\
N (Locoregional lymph node) & 12 \\
M (Extraregional Lymph node, bone) & 1 \\
Local recurrence & \\
\hline
\end{tabular}

TCC - transitional cell cancer; RT - radiotherapy; KPS - Karnofsky performance status.

Table 2. Site and size of the treated lesions.

\begin{tabular}{llc}
\hline $\begin{array}{l}\text { Number of } \\
\text { lesions }\end{array}$ & Site of the lesion & Size of the lesion $\left[\mathbf{c m}^{3}\right]$ \\
\hline 10 & Pelvic lymph-node & $44.5(7.3-127.7)^{*}$ \\
5 & Para/Lombo-aortic lymph-node & $3.9(2.9-25.8)^{*}$ \\
1 & Para-vaginal lymph-node & 31.8 \\
1 & Supra-clavicular lymph-node & 16.3 \\
1 & Para-vertebral lymph-node & 7.0 \\
1 & Lung lesion & 3.0 \\
1 & Pelvic recurrence & 45.0 \\
1 & Pelvic bone & 88.7 \\
\hline
\end{tabular}

${ }^{*}$ Median and range values are presented.

Table 3. Treatment schemes.

\begin{tabular}{lcccc}
\hline $\begin{array}{l}\text { Number of } \\
\text { patients }\end{array}$ & $\begin{array}{c}\text { Number of } \\
\text { fractions }\end{array}$ & $\begin{array}{c}\text { Dose per } \\
\text { fraction [Gy] }\end{array}$ & $\begin{array}{c}\text { BED } \\
(\boldsymbol{\alpha} / \boldsymbol{\beta}=\mathbf{1 0} \text { Gy) [Gy] }\end{array}$ & $\begin{array}{c}\text { EQD2 } \\
{[\mathbf{G y}]}\end{array}$ \\
\hline 12 & 5 & 5 & 37.5 & 31.25 \\
3 & 8 & 3 & 31.2 & 26.0 \\
1 & 5 & 4 & 28.0 & 23.33 \\
1 & 4 & 5 & 30.0 & 25.0 \\
1 & 5.7 & 5 & 42.75 & 35.63 \\
1 & 6 & 5 & 45.0 & 37.5 \\
1 & 12 & 3 & 46.8 & 39.0 \\
1 & 3 & 10 & 60.0 & 50.0 \\
\hline
\end{tabular}

Gy - gray; BED - biologically effective dose; EQD2 - 2-Gy fraction equivalent dose.
Committee notification regarding the research on SBRT (notification Nr. 93/11).

\section{Results}

Patients. Thirteen TCC metastatic patients underwent RT for 21 lesions in our Institution between December 2012 and November 2017. The characteristics of the 13 patients included in this study are summarized in Table 1 . The mean age at SBRT was years 68 years (range 50-80 years) and Karnofsky performance status (KPS) was 90 (range 80-90). Median interval between TCC diagnosis and the start of RT on oligometastasis was 23 months (range 8-105).

Treatment included irradiation of one or more lymph node metastasis sites for 10 patients: 6 patients were treated for one lesion and 4 patients received RT for more than one lesion (2-4) concomitantly. One patient was treated with several RT treatments (metachronous SBRT) for different lymph node recurrences within a period of 12 months. Two patients were treated for single bone and lung metastasis, respectively. The site and size of treated lesions is presented in Table 2.

Patients treatment. The patient with bone metastases was treated with intensity modulated RT (IMRT) using ExacTrac $^{\circledast}$ system (BrainLAB AG, Germany) for imageguidance. The dose prescribed to PTV was $20 \mathrm{~Gy}$ in 4 fractions. The patient with lung metastases was treated with CyberKnife (Accuray Inc., Sunnyvale, USA) and the dose prescribed to PTV was $36 \mathrm{~Gy}$ in 3 fractions. One patient was treated for local recurrence.

The total RT doses ranged from 20 to $36 \mathrm{~Gy}$, given in 3 to 10 fractions (median $25 \mathrm{~Gy} / 5$ fractions), with median BED 37.5 Gy (range 28-60 Gy) and median EQD2 31.25 Gy (range $23.3-50 \mathrm{~Gy}$ ), considering $\alpha / \beta$ ratio of $10 \mathrm{~Gy}$. Detail about treatment schemes are presented in Table 3. Median follow-up was of 25 months (range 3-43 months).

The results of the first radiological evaluation (3 months after RT) of the 21 lesions are summarized in Table 4 . The median interval between the completion of RT and a first in-field progression was 5.8 months (2.3-13.4 months). The clinical out- and in-field progression of the disease was registered in 9 patients at 4.2 months (median value, range 1.9-18.8 months) from the end of RT and detected by radiological imaging; in 6 cases it was both out- and in-field progression while in 3 patients we registered an out-field progression only. One of the patients with out-field progression underwent a chemotherapy with Carboplatin and Gemcitabine (CBDCA/GEM) cycle with complete response at the last clinical evaluation.

At last contact, 3 patients are disease-free, 3 are alive with disease, 6 died of TCC and one died for other disease (Table 4). RT was very well tolerated. According to RTOG/ EORTC criteria [23], only one patient developed acute grade 1 GI toxicity. No late grade $>2$ adverse events were observed during the follow-up period. 


\section{Discussion}

The study, including 13 patients/21 lesions treated with high precision RT, showed that such approach is feasible, very well tolerated and offers complete tumor remission in about a third of the cases at 25-month follow-up.

Since the definition of oligomestatic disease [25], this concept has revolutionized the clinical practice, establishing for local treatment a new role in the context of an assumed systemic disease. Its relevance is based on the hypothesis that localized forms of cancer treatment may be effective in patients with oligometastases. Nowadays the definition of oligometastases is not consensual both in terms of clinical presentation and of the biological basis [26] and it appears even more complicated in bladder cancer. Oligometastatic status implies oligoprogression, namely a temporarily transitory condition where the eradication of an important part of macroscopic disease can give an advantage to prolong the survival. Systemic chemotherapy is the standard of care for metastatic bladder disease, no matter if in presence of a polimetastatic or oligometastatic. RT was introduced as an option to face challenges such as local relapse or oligometastases in the treatment of numerous tumor diseases such as prostate, breast lung, kidney and colorectal cancer [11-17]. Advances in multimodal treatment strategies involving all major oncologic disciplines have contributed to a remarkably improved prognosis for oligometastatic patients. Navarria et al. reported the results of SBRT in lung oligometastatic patients from different primary tumors resulting in a 3-year overall survival of $73 \%$ [24].

Single case reports and series of SBRT for primary TCC and renal cell carcinoma of kidney show very promising results [19-20], but there are no consistent data in literature about the oligometastatic/oligorecurrent setting of TCC. We retrospectively evaluated our small series of patients in order to investigate the possible role of irradiation using high precision technologies in the treatment of oligometastatic/ oligorecurrent bladder cancer patients. We aimed to evaluate the disease free interval before a further progression and consequently the starting of a new systemic treatment. The primary intent in our study was to report on local control and induced toxicity in patients with TCC oligometastasis treated with RT. Obviously, we keep in mind the main limits of this study, namely the retrospective character, the small number of our patient series and the non-homogeneity of the RT techniques. However, our results compare well to the "standard therapy" reports on metastatic TCC.

Reports on consolidative RT after chemotherapy for metastatic TCC cancer also demonstrate that radiation is feasible and might contribute to long-term disease control [27]. The employment of stereotactic treatment in conjunction with hypofractionation might be inappropriate if the $\alpha / \beta$ ratio of TCC is very high (reported in a range of 10-30 Gy). On the other hand, recent data suggest the possibility of a low $\alpha / \beta$ ratio, so hypofractionation may be a reasonable choice in this setting [28]. Furthermore, reduction in the number of fractions allows a better compliance to treatment in this cohort of patients, generally largely pre-treated. Most of the patients included in the study were previously chemo-treated and/or underwent multiple surgical procedures; this made them not easily candidates for undergoing other invasive and debilitating treatments. Radiotherapy offers a less invasive treatment, better tolerated, allowing an ablation of the treated metastasis in most cases.

Furthermore, we observed a very low toxicity profile, and one third of the patients reach the aim of disease free condition at 30 months after RT, without any other adjunctive systemic treatment. However, considering the consistent rate of in-field progression, the absence relevant toxicity and the average delivered dose that can almost be considered "palliative", the necessity of dose escalation is urgent. Also we have to face the problem of the systemic evolution in this set of patients, but the natural history of the disease of our series of patients shows that the further progression frequently appear as oligorecurrence: so the corner point is to understand how select the patients that could really benefit of local therapy approach. The identification of predictive prognostic biomarkers is a further step forward in patient stratification in order to distinguish real oligometastatic patients from polymetastatic patients and to offer a personalized treatment.

More effective treatments are needed: new landscape is offered by immunotherapy including pembrolizumab or atezolimumab, recently approved in metastatic bladder cancer. Combination of treatments should be investigated. Interestingly, RT showed a stimulation of the immune system, therefore the future investigation regarding the optimal combination of these treatments is warranted. Our study suggests that limited metastatic TCC disease can be safely and effectively treated with targeted RT. In this series, 3 patients are free of disease at 25 months. Ablative-intent RT could be considered for selected patients with oligo-recurrence, unfit to or refusing a systemic therapy.

Table 4. Follow-up data.

\begin{tabular}{lc}
\hline First radiological response to RT (per lesion) & N (\%) \\
\hline CR & $11(52)$ \\
PR & $1(5)$ \\
SD & 0 \\
LPD & $8(38)$ \\
NE & $1(5)$ \\
\hline Status at last contact (per patient) & $3(23)$ \\
\hline Alive without disease & $3(23)$ \\
Alive with disease & $6(46)$ \\
Died due to disease & $1(8)$ \\
Lost to follow-up
\end{tabular}

CR - complete response; PR - partial response; SD - stable disease; LPD local progression disease; NE - not evaluable; RT - radiotherapy. 


\section{References}

[1] ROSENBERG JE, CARROLL PR, SMALL EJ. Update on chemotherapy for advanced bladder cancer. J Urol 2005;174: 14-20. https://doi.org/10.1097/01.ju.0000162039.38023.5f

[2] MAFFEZZINI M, AUDISIO R, PAVONE-MACALUSO M, HALL RR. Bladder cancer. Crit Rev Oncol Hematol 1998; 27: 151-153. https://doi.org/10.1016/S1040-8428(97)10026-9

[3] MOSCHINI M, KARNES RJ, SHARMA V, GANDAGLIA G, FOSSATI $N$ et al. Patterns and prognostic significance of clinical recurrences after radical cystectomy for bladder cancer: A 20-year single center experience. Eur J Surg Oncol 2016; 42: 735-743. https://doi.org/10.1016/j.ejso.2016.02.011

[4] EDGE S, BYRD DR, COMPTON CC, FRITZ AG, GREENE F et al (Eds.). AJCC Cancer Staging Manual, 7th Edition. Springer-Verlag, New York 2010, p 718. ISBN 978-0-38788442-4

[5] YAFI FA, APRIKIAN AG, CHIN JL, FRADET Y, IZAWA $J$ et al. Contemporary outcomes of 2287 patients with bladder cancer who were treated with radical cystectomy: a Canadian multicentre experience. BJU Int 2011; 108: 539-545. https://doi.org/10.1111/j.1464-410X.2010.09912.x

[6] LOGOTHETIS CJ, DEXEUS F, FINN L, SELLA A, AMATO $\mathrm{RJ}$ et al. A prospective randomized trial comparing CISCA to MVAC chemotherapy in advanced metastastic urothelial tumors. J Clin Oncol 1990; 8: 1050-1055. https://doi. org/10.1200/JCO.1990.8.6.1050

[7] ROSENBERG JE, HOFFMAN-CENSITS J, POWLES T, VAN DER HEIJDEN MS, BALAR AV et al. Atezolizumab in patients with locally advanced and metastatic urothelial carcinoma who have progressed following treatment with platinum-based chemotherapy: a single-arm, multicentre, phase 2 trial. Lancet 2016; 387: 1909-1920. https://doi. org/10.1016/S0140-6736(16)00561-4

[8] BALAR AV, GALSKY MD, ROSENBERG JE, POWLES T, PETRYLAK DP et al. Atezolizumab as first-line treatment in cisplatin-ineligible patients with locally advanced and metastatic urothelial carcinoma: a single-arm, multicentre, phase 2 trial. Lancet 2017; 389: 67-76. https://doi.org/10.1016/ S0140-6736(16)32455-2

[9] BALAR A, BELLMUNT J, O'DONNELL PH, CASTELLANO D, GRIVAS P et al. Pembrolizumab (pembro) as first-line therapy for advanced/unresectable or metastatic urothelial cancer: preliminary results from the phase $2 \mathrm{KEY}$ NOTE 052 study. Ann Oncol 2016; 27: vi552-vi587 (Suppl 6). https://doi.org/10.1093/annonc/mdw435.25

[10] BURGER M, CATTO JW, DALBAGNI G, GROSSMAN HB, HERR $H$ et al. Epidemiology and risk factors of urothelial bladder cancer. Eur Urol 2013; 63: 234-241. https://doi. org/10.1016/j.eururo.2012.07.033

[11] DE RUYSSCHER D, WANDERS R, VAN BAARDWIJK A, DINGEMANS AM, REYMEN B et al. Radical treatment of non-small-cell lung cancer patients with synchronous oligometastases: long-term results of a prospective phase II trial (Nct01282450). J Thorac Oncol 2012; 7: 1547-1555. https:// doi.org/10.1097/JTO.0b013e318262caf6
[12] ASHWORTH AB, SENAN S, PALMA DA, RIQUET M, AHN YC et al. An individual patient data metaanalysis of outcomes and prognostic factors after treatment of oligometastatic non-small-cell lung cancer. Clin Lung Cancer 2014; 15: 346-355. https://doi.org/10.1016/j.cllc.2014.04.003

[13] WANG HH, ZAORSKY NG, MENG MB, ZENG XL, DENG $\mathrm{L}$ et al. Stereotactic radiation therapy for oligometastases or oligorecurrence within mediastinal lymph nodes. Oncotarget 2016; 7: 18135-18145. https://doi.org/10.18632/oncotarget.7636

[14] KANG JK, KIM MS, KIM JH, YOO SY, CHO CK et al. Oligometastases confined one organ from colorectal cancer treated by SBRT. Clin Exp Metastasis 2010; 27: 273-278. https://doi.org/10.1007/s10585-010-9325-0

[15] JERECZEK-FOSSA BA, PIPERNO G, RONCHI S, CATALANO G, FODOR C et al. Linac-based stereotactic body radiotherapy for oligometastatic patients with single abdominal lymph node recurrent cancer. Am J Clin Oncol 2014; 37: 227-233. https://doi.org/10.1097/COC.0b013e3182610878

[16] JERECZEK-FOSSA BA, BELTRAMO G, FARISELLI L, FODOR C, SANTORO L et al. Robotic image-guided stereotactic radiotherapy, for isolated recurrent primary, lymph node or metastastic prostate cancer. Int J Radiat Oncol Biol Phys 2012; 82: 889-897. https://doi.org/10.1016/j. ijrobp.2010.11.031

[17] JERECZEK-FOSSA BA, BOSSI-ZANETTI I, MAURO R, BELTRAMO G, FARISELLI L et al. CyberKnife robotic image-guided stereotactic radiotherapy for oligometastic cancer: A prospective evaluation of 95 patients/118 lesions. Strahlenther Onkol 2013; 189: 448-455. https://doi. org/10.1007/s00066-013-0345-y

[18] RODRÍGUEZ-RUIZ ME, SAN MIGUEL I, GIL-BAZO I, PEREZ-GRACIA JL, ARBEA L et al. Pathological vertebral fracture after stereotactic body radiation therapy for lung metastases. Case report and literature review. Radiat Oncol 2012; 7: 50. https://doi.org/10.1186/1748-717X-7-50

[19] KOLLA SB, HEMAL AK. An unusual case of transitional cell carcinoma of renal pelvis presenting with brain metastases. Int Urol Nephrol 2007; 39: 747-750. https://doi.org/10.1007/ s11255-006-9155-9

[20] MOON KS, JUNG S, LEE KH, HWANG EC, KIM IY. Intracranial metastasis from primary transitional cell carcinoma of female urethra: case report \& review of the literature. BMC Cancer 2011; 11: 23. https://doi.org/10.1186/1471-2407-1123

[21] TIMMERMAN RD. An overview of hypofractionation and introduction to this issue of seminars in radiation oncology. Semin Radiat Oncol 2008; 18: 215-222. https://doi. org/10.1016/j.semradonc.2008.04.001

[22] THERASSE P, ARBUCK SG, EISENHAUER EA, WANDERS J, KAPLAN RS et al. New Guidelines to Evaluate the Response to Treatment in Solid Tumors. J Natl Cancer Inst 2000; 92: 205-216.

[23] COX JD, STETZ J, PAJAK TF. Toxicity criteria of the Radiation Therapy Oncology Group (RTOG) and the European Organization for Research and Treatment of Cancer (EORTC). Int J Radiat Oncol Biol Phys 1995; 31: 1341-1346. https://doi.org/10.1016/0360-3016(95)00060-C 
[24] NAVARRIA P, ASCOLESE AM, TOMATIS S, COZZI L, DE ROSE F et al. Stereotactic body radiotherapy (SBRT) in lung oligometastatic patients: role of local treatments. Radiat Oncol 2014; 9: 91. https://doi.org/10.1186/1748-717X-9-91

[25] HELLMAN S, WEICHSELBAUM RR. Oligometastases. J Clin Oncol 1995; 13: 8-10. https://doi.org/10.1200/ JCO.1995.13.1.8

[26] THARIAT J, VIGNOT S. Les concepts d'oligométastase et d’oligoprogression. Bull Cancer 2016; 103: S48-S54. https:// doi.org/10.1016/S0007-4551(16)30145-X
[27] SHAH S, ZHANG CA, HANCOCK S, FAN A, SKINNER E et al. Consolidative Radiotherapy in Metastatic Urothelial Cancer. Clin Genitourin Cancer 2017: 15: 685-688. https:// doi.org/10.1016/j.clgc.2017.04.007

[28] KANG JJ, IWAMOTO KS, PEEK EM, CHIN AI, KING CR. The Low Alpha-Beta Ratio of Bladder Cancer: A Rationale for Hypofractionation. Proceedings of the 96th Annual Meeting of the American Radium Society 2014. http://www. cancernetwork.com/ars-2014/low-alpha-beta-ratio-bladdercancer-rationale-hypofractionation [accessed online 05-072018]. 